\title{
Shin Splints: Efficacy of Radial Extracorporeal Shock Wave Therapy in Ballet Dancers. A Series Of Cases
}

\author{
Tutté ML, ${ }^{1,2,3} \mathrm{PhD}$; Galin $\mathrm{G}^{1,2} \mathrm{PT}$ \\ ${ }^{I}$ (Department of Rehabilitation, Central de Servicios Médicos, Banco de Seguros del Estado, Montevideo, \\ Uruguay) \\ ${ }^{2}$ (Department of Rehabilitation, Ondas de Choque Uruguay, Montevideo, Uruguay) \\ ${ }_{3}^{3}$ (Department of Rehabilitation, Blue Cross \& Blue Shield, Montevideo, Uruguay)
}

\begin{abstract}
Shin splints - also known as medial tibial stress syndrome, anterior compartment and posteromedial compartment syndrome - have been widely studied in athletes, mostly in runners, but not in dancers. This paper presents the results of treatment with radial extracorporeal shockwave therapy (rESWT) in 4 ballet dancers with shin splint diagnosis. Four patients were treated with two different rESWT devices after the failure of different therapeutic modalities. They received 6 weekly sessions applied along the tibial shaft $(3,000 \mathrm{p}, 2.5 \mathrm{~b}, 7 \mathrm{~Hz})$, and a stretching program. The Visual Analogue Scale (VAS) was used for pain quantification during ballet training and a rating score to determine maximum painless jumping tolerance. Mean decrease in pain VAS score was 4,8 points. Impairment of exercise tolerance was reduced from 3 to 1 points. Patients were able to continue with their normal training and performance schedules. Three were asymptomatic after 6 months; one experienced some discomfort during high-intensity rehearsals, but was still able to perform. rESWT was shown to be an effective, non-invasive treatment alternative for these patients, achieving functional improvement and pain relief, allowing them to comply with their performance schedules. Large-scale prospective trials are still required to elucidate the effectiveness of $r E S W T$ in these patients.
\end{abstract}

Keywords: dancers, medial tibial stress syndrome, shin splints, shock wave therapy.

\section{Introduction}

In 1966, the term "shin splints" was defined by the American Medical Association in the Standard Definition of Athletic Injuries as follows: "pain and discomfort in the leg from repetitive running on hard surfaces or forcible extensive use of flexors..." [1]. Various names have been introduced for the complex of this kind of musculoskeletal disorder: medial tibial syndrome, medial tibial stress syndrome (MTSS) [2], shin soreness [3], anterior compartment syndrome [1], and posteromedial compartment syndrome [1].

As regards to etiology, a suggested mechanism is an overcharge of the anterior compartment of the leg muscles, fascias and osseas and periostical tibial traction of the soleus or the long toes flexor muscle [4]. However, the hypothesis of periostitis has not yet been corroborated by a histological correlate. Beck [5] and Moen [6] described the theory that it is caused by bony resorption that outpaces bone formation of the tibial cortex. De Lacerda [7] found shin splints to be related to foot pronation.

This pathology has been studied in athletes, most of them runners. Very few current studies exist of shin splints in ballet dancers [8], and even less about treatment with rESWT in these patients. Although shin splints may be caused by several factors common to runners and dancers, a common theory among ballet dancers and teachers is that shin splints result from jumping without placing the heel on the floor upon landing. From antique cinematographic studies, Kantor [9] revealed that runners with a history of shin splints never made heel contact on the initial return to the ground. One possible cause for lifting the heel could be a tight Achilles tendon. Another contributor factor is running or jumping on hard surfaces. [7].

Symptoms are usually experienced during sports activities, and sometimes even when walking without having any trauma. In this kind of patients, history often includes a recent change or increase in physical activity or repetitive exercise with minimal recovery time. Patients recount pain along tibial shaft and pain with percussion during weight-bearing activity. Pain subsides in periods of low physical stress but relapses when athletes do sports again [10]. Shin-splint pain has two common sites. Runners usually feel pain along the $2 / 3$ distal posteromedial aspect of the lower leg in an area posterior to the medial malleolus. The other pain site is along the anterolateral aspect of the lower leg and along the proximal portion of the tibialis anterior $[4,8]$. This site is most common among ballet dancers [8].

If not treated properly, this pathology can be quite disabling and progress to more serious complications, like tibial stress fractures [11-13]. 


\section{Material And Methods}

Four ballet dancers with shin splint diagnosis were treated with 2 different devices of rESWT after the fail of different therapeutic modalities. Three of them were men (20, 22 and 23 years old), and 1 woman (15 years old). Two of them were treated with an EMS Swiss Dolorclast ${ }^{\circledR}$ Classic, and 2 of them with LONGEST PowerShocker LGT-2500S.

Case 1. Twenty-year-old male with a 2-year history of pain, exacerbated in the past 4 months concurrently with an increase in physical activity level. Describes bilateral pain along the entire tibial shaft, predominantly along the 2/3 distal posteromedial aspect of the lower leg. Radiography showed multiple radiolucid areas of cortical bone in tibial diaphysis, with a marked left side predominance in the anterior section. (Fig. 1a. 1b.). MRI showed periosteal oedema only. (Grade1, Fredericson classification for tibial stress fractures) [14].

Case 2. Twenty-two-year-old male with a 1-year history of pain, describes focal pain along left anterolateral tibial shaft, predominantly proximal, during weight-bearing activity with knee flexion (plié) and with jumps. String-of-beads pattern is perceived along the tibial shaft upon palpation.

Case 3. Twenty-three-year-old male, recounts similar symptoms as in Case 2.

Case 4. Fifteen-year-old female student of the National Ballet School, with a 6-month history of bilateral pain along the tibial shaft that prevent her from finishing ballet classes. Pronated right foot.

They received 6 weekly sessions, with $3000 \mathrm{p}, 2,5 \mathrm{~b}, 7 \mathrm{~Hz}$ per session protocol, applied along the tibial shaft (pain zone), combined with a program of stretching exercises and counseling on correct jumping technique. 2 patients had bilateral pain, totaling 6 observations. The Visual Analogue Scale (VAS) was used for the quantification of pain during ballet training, concretely during jumping. Another rating score, proposed by Schulze [15], was used to determine maximum painless tolerance when jumping, where: $0=$ no pain when jumping; 1 = slight pain; 2 = severe pain when jumping; 3 = jumping impossible.

\section{Results}

Mean decrease in pain with VAS score was 4,8 points at 2 months from baseline. (4,7 with EMS device and 5 with LONGEST device). Impairment of exercise tolerance was reduced from 3 to 1 points. Patients were able to continue with their normal training and performance schedules. Three of them were asymptomatic after 6 months; one experienced some discomfort during high-intensity rehearsals, but was still able to perform.

\section{Discussion}

The studied cases show that the rESWT therapy is a potentially effective method for treatment of shin splints in dancers, achieving functional improvement and pain relief. This treatment, applied properly, could prevent serious complications like the tibial stress fracture. It must not be forgotten that an important part of the treatment is to evaluate a dancer's jumping technique and provide systematic training to develop the skill and avoid hard surfaces. Stretching exercise for Achilles tendon when it is tight are also required.

As said before, there are very few studies of shin splints in ballet dancers, and even less about the treatment with radial ESWT in this kind of patients. We found only one article referred to shin splints in ballet dancers [8]; the hypothesis of the study was that dancers with a history of recurring shin splints, manifested as pain along the tibia, do not place their heels on the floor as often as ballet dancers without such a history. The usual technique of classical ballet jumping begins with a knee bend with the heels on the floor, the weight is then transferred from the heels to the ball of the foot while the knees and hips begin to extend. The final pushoff is given by the toes. At the peak of the jump, the knees are extended and the ankles are strongly plantar flexed. In the first stage of landing, the toes and then the ball of the foot contact the floor and continue to a more complete landing by placing the heel on the floor. They complete the jump in a knee bend like the initial position. The next jump can then be initiated from this position. Some dancers land from one jump and push off for the next separately by a brief instant where the heel pops off the floor, and the author found that this situation had a significant relationship ( $\mathrm{p} .<0.05)$ with a history of shin splints. Although this study had a very small $\mathrm{N}$ (16 dancers) and used simple and now outdated equipment, it was among the first investigations into the etiology of shin splints in ballet dancers.

A search conducted in PubMed using the keywords "shin splints" OR "Medial tibial stress syndrome" AND "shock wave therapy" yielded only two studies. One of them, by Rompe et al, [16], was a cohort study of 47 subjects with chronic recalcitrant MTSS that underwent a standardized home training program and received repetitive low-energy rESWT (2000 shocks; 2.5 bars of pressure), and a control group of 47 subjects who only received the same training program. They found that at 15 months from baseline, 40 of 47 subjects in the treatment group had been able to return to their preferred sport at their preinjury level, as had 22 of the 47 control subjects, so the conclusion was that rESWT as applied was an effective treatment for MTSS.

The other one study, by Moen et al [17], was a prospective controlled trial of 42 athletes with MTSS, performed with focused ESWT. One group was treated with a graded running programme, while patients in the other group were treated with the same graded running programme and focused ESWT (five sessions in 9 
weeks).The study showed that MTSS patients may benefit from ESWT in addition to a graded running programme.

Although there is abundant literature about "shin splints" AND "running", a similar search "dancers" didn't yield any results. For this reason, we believe that the cases described in this paper, though just a few, may constitute an interesting contribution, even if large, controlled studies are still required.

\section{Conclusion}

Both rESWT devices were shown to be an effective, non-invasive treatment alternative for these patients, achieving functional improvement and pain relief and allowing them to comply with their performance schedules. Large-scale prospective trials are still required to elucidate the effectiveness of ESWT for shin splints in ballet dancers.

\section{Acknowledgements}

Thanks to Andrea Tutté and Leonardo Giangreco, who assisted in the preparation of this manuscript.

\section{Disclosure}

The authors report no conflicts of interest in this work.

Figure 1 Multiple radiolucid areas of cortical bone in anterior tibial diaphysis, a) proximal b) distal tibial shaft.
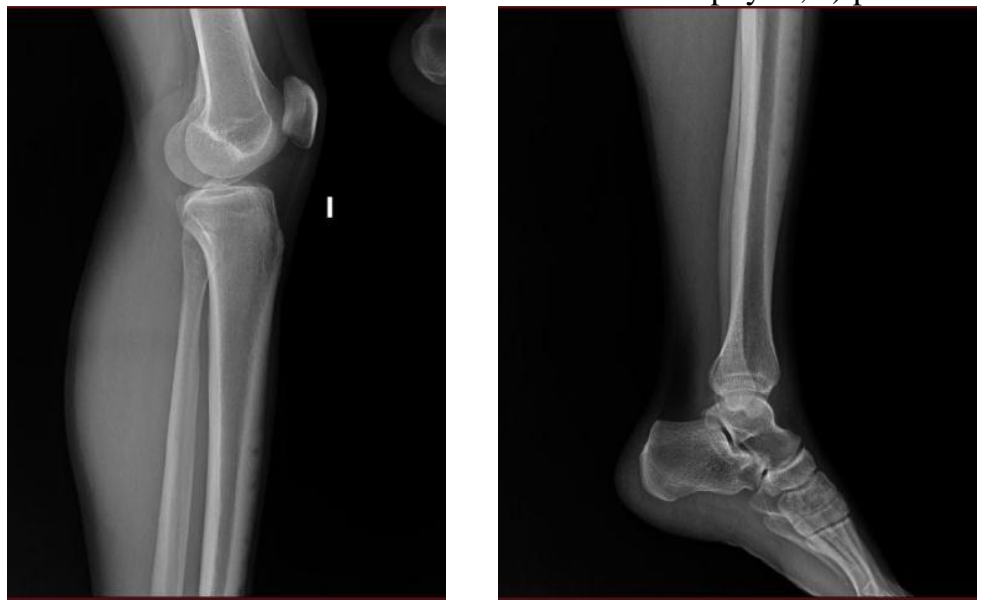

\section{References}

[1]. P. Bates et al. Shin splints: a literature review, British Journal of Sports Medicine, 19(3) 1985, $132-137$.

[2]. S. J. Mubarak et al. The medial tibial stress syndrome. A cause of shin splints, The American Journal of Sports Medicine, 10(4), 1982, 201-205.

[3]. M. B. Devas et al. Stress fractures of the tibia in athletes or shin soreness, The Journal of Bone and Joint Surgery, 40-B(2), 1958 $227-239$.

[4]. S. B. Brotzman. Clinical Orthopaedic Rehabilitation, (Second edition. Ed. Elsevier, 2005)

[5]. B. R. Beck et al. Tibial stress injuries. An etiological review for the purposes of guiding management, Sports Medicine, 26(4), 1998, 265-279.

[6]. M. H. Moen et al. Medial tibial stress syndrome: a critical review, Sports Medicine, 39(7),2009, 523-546.

[7]. F. G. DeLacerda et al. The study of anatomical factors involved in shin splints, Journal of Orthopaedic and Sports Physical Therapy $2,1980,55-59$

[8]. A. Gans. The Relationship of Heel Contact in Ascent and Descent from Jumps to the Incidence of Shin Splints in Ballet Dancers, Phys Ther, 65, 1985, 1192-1196.

[9]. L. A. Kantor, Survey of the Etiology of Shin Splints, Thesis, Springfield College, Springfield, MA, 1948.

[10]. B. Yates, S. White, The incidence and risk factors in the development of medial tibial stress syndrome among naval recruits, The American Journal of Sports Medicine, 32(3), 2004, 772-780.

[11]. L. Kahanov et al. Review: Diagnosis, treatment, and rehabilitation of stress fractures in the lower extremity in runners, Open Access Journal of Sports Medicine, 27 march 2015.

[12]. M. Fredericson et al. Stress fracture in athletes, Top Magn Reson Imaging., 17(5), 206, 309-325.

[13]. K. G. Kilcoyne et al. Tibial stress fractures in an active duty population: long-term outcomes, J Surg Orth Adv, $22(1), 2013,50-53$.

[14]. Kijowski R. et-al. Validation of MRI classification system for tibial stress injuries, AJR Am J Roentgenol.198 (4), $2012,878-84$.

[15]. Schulze C et al. Treatment of Medial Tibial Stress Syndrome according to the Fascial Distortion Model: A Prospective Case Control Study, Hindawi Publishing Corporation. The Scientific World Journal, 2014, Article ID 790626, 6 pages http://dx.doi.org/10.1155/2014/790626.

[16]. Rompe J. D. et al. Low-energy extracorporeal shock wave therapy as a treatment for medial tibial stress syndrome, Am J Sports Med. 38(1), 2010, 125-32.

[17]. Moen M. H. et al. Shockwave treatment for medial tibial stress syndrome in athletes; a prospective controlled study, British Journal of Sports Medicine, 46(4), 2012, 253-257. 